\title{
Refractory Colon Carcinoma
}

National Cancer Institute

\section{Source}

National Cancer Institute. Refractory Colon Carcinoma. NCl Thesaurus. Code C153351.

Colon carcinoma that is resistant to treatment. 